PROCEEDINGS OF THE

AMERICAN MATHEMATICAL SOCIETY

Volume 131, Number 3, Pages 685-699

S 0002-9939(02)06562-0

Article electronically published on July 17, 2002

\title{
MODULI OF TWISTED SPIN CURVES
}

\author{
DAN ABRAMOVICH AND TYLER J. JARVIS
}

(Communicated by Michael Stillman)

\begin{abstract}
In this note we give a new, natural construction of a compactification of the stack of smooth $r$-spin curves, which we call the stack of stable twisted $r$-spin curves. This stack is identified with a special case of a stack of twisted stable maps of Abramovich and Vistoli. Realizations in terms of admissible $\mathbb{G}_{\mathbf{m}}$-spaces and $\mathbb{Q}$-line bundles are given as well. The infinitesimal structure of this stack is described in a relatively straightforward manner, similar to that of usual stable curves.

We construct representable morphisms from the stacks of stable twisted $r$-spin curves to the stacks of stable $r$-spin curves and show that they are isomorphisms. Many delicate features of $r$-spin curves, including torsion free sheaves with power maps, arise as simple by-products of twisted spin curves. Various constructions, such as the $\bar{\partial}$-operator of Seeley and Singer and Witten's cohomology class go through without complications in the setting of twisted spin curves.
\end{abstract}

The moduli space of smooth $r$-spin curves was compactified by the second author, using torsion free sheaves and coherent nets of torsion free sheaves in [4] and [5]. In order to construct a satisfactory compactification it was necessary in those papers to study in detail the behavior of torsion free sheaves with an $r$-power map. The infinitesimal properties of those compactifications are quite subtle.

The purpose of this note is to give a new, natural construction of a compactification of the stack of smooth $r$-spin curves, which we call the stack of stable twisted $r$-spin curves, and to describe its properties and relations to other compactifications.

In the first section we define the stack of smooth $r$-spin curves and give an alternate construction in terms of certain principal $\mathbb{G}_{\mathbf{m}}$-bundles. To generalize these to stable curves we then recall the twisted curves of [1] and give a natural definition of twisted stable $r$-spin curves, very much analogous to the situation of 2. We show that over smooth curves all of these constructions are equivalent to each other. Moreover, we identify the stack of twisted stable $r$-spin curves with a special case of a stack of twisted stable maps of [1].

In the second section we describe the infinitesimal structure of this stack, again in analogy to the treatment of 2. This is relatively straightforward and is similar to the infinitesimal structure of usual stable curves.

Received by the editors April 13, 2001 and, in revised form, October 11, 2001.

2000 Mathematics Subject Classification. Primary 14H10.

The first author's research was partially supported by NSF grants DMS-9700520 and DMS0070970.

The second author's research was partially supported by NSA grant MDA904-99-1-0039.

(C)2002 American Mathematical Society 
In the third and fourth sections we discuss more details of the various characterizations in the non-smooth case. In the third section we describe certain admissible $\mathbb{G}_{\mathbf{m}}$-spaces that correspond to twisted stable spin curves. In the fourth section we describe the compactifications of [4] and [5] of the stack of stable $r$-spin curves, using coherent nets of rank-one torsion free sheaves, and we construct representable morphisms from the stack of stable twisted $r$-spin curves to those compactifications, which enables us to compare them. It turns out that our stack of twisted spin curves is isomorphic to that of coherent nets of roots introduced in [5. Many of the delicate features of coherent nets of torsion free sheaves arise as simple by-products of twisted spin curves.

In the fifth section we discuss the appropriate generalization of Witten's cohomology class 11 to the setting of twisted spin curves. A key element of Witten's construction is the $\bar{\partial}$-operator of Seeley and Singer [10], and we discuss how the $\bar{\partial}$-operator may be generalized to the case of twisted spin curves.

\section{Spin CURVES AND TWISTED SPIN CURVES}

We fix an integer $r>0$, and throughout this paper we will consider only schemes over Spec $\mathbb{Z}[1 / r]$.

1.1. Moduli of smooth $r$-spin curves. Let $g$ and $n$ be integers such that $2 g-2+$ $n>0$. Let $\mathbf{m}=\left(m_{1}, \ldots, m_{n}\right)$ be an $n$-tuple of integers. A non-singular $n$-pointed $r$-spin curve of genus $g$ and type $\mathbf{m}-\mathbf{1}$ over a scheme $S$, denoted $\left(C \rightarrow S, s_{i}, \mathcal{L}, c\right)$, is the data of

(1) a smooth, $n$-pointed curve $\left(C \rightarrow S, s_{i}: S \rightarrow C\right)$ of genus $g$,

(2) an invertible sheaf $\mathcal{L}$ on $C$, and

(3) an isomorphism $c: \mathcal{L}^{\otimes r} \stackrel{\sim}{\longrightarrow} \omega_{C / S}\left(\sum_{i=1}^{n}\left(1-m_{i}\right) S_{i}\right)$ where $S_{i}$ is the image of $s_{i}$.

In simple terms, a non-singular $r$-spin curve of type $\mathbf{m}-\mathbf{1}$ is a pointed curve with the choice of an $r$ th root of the sheaf of logarithmic 1-forms which vanish to order $m_{i}$ along the $i$ th section. We should point out that, while the definition given here in terms of logarithmic differentials vanishing to order $\mathbf{m}$ is the most convenient to work with when studying twisted spin curves (due to invariance of the generators $d z / z)$, the equivalent definition in terms of regular differentials vanishing to order $\mathbf{m} \mathbf{- 1}$ is more common (compare, for example, [5] 11]).

A morphism from one $r$-spin curve $\left(C^{\prime} \rightarrow S^{\prime}, s_{i}^{\prime}, \mathcal{L}^{\prime}, c^{\prime}\right)$ to another $\left(C \rightarrow S, s_{i}, \mathcal{L}, c\right)$ consists of a fiber diagram $(\phi, \alpha)$, i.e., an $S$-morphism $\phi: C^{\prime} \rightarrow C$, inducing an isomorphism $C^{\prime} \rightarrow C \times{ }_{S} S^{\prime}$,

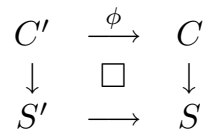

along with an isomorphism $\alpha: \mathcal{L}^{\prime} \rightarrow \phi^{*} \mathcal{L}$, such that $\phi^{*} c \circ \alpha^{\otimes r}=c^{\prime}$.

The category $\mathcal{M}_{g, n}^{1 / r, \mathbf{m}-\mathbf{1}}$ of non-singular, $n$-pointed $r$-spin curves of genus $g$ and type $\mathbf{m}-\mathbf{1}$, with morphisms given by fiber diagrams, is a Deligne-Mumford stack with quasi-projective coarse moduli space $\mathbf{M}_{g, n}^{1 / r, \mathbf{m}-\mathbf{1}}$. See [5] for a detailed proof. When $\mathbf{k}$ is congruent to $\mathbf{k}^{\prime} \bmod r$, the two stacks $\mathcal{M}_{g, n}^{1 / r, \mathbf{k}}$ and $\mathcal{M}_{g, n}^{1 / r, \mathbf{k}^{\prime}}$ are canonically isomorphic. We denote by $\mathcal{M}_{g, n}^{1 / r}$ the disjoint union $\coprod_{-1 \leq k_{i}<r-1} \mathcal{M}_{g, n}^{1 / r, \mathbf{k}}$. 
Note that if $\left(C \rightarrow S, s_{i}, \mathcal{L}, c\right)$ is a non-singular, $n$-pointed $r$-spin curve of genus $g$ and type $\mathbf{m}-\mathbf{1}$, and $S$ is the prime spectrum of a field, then

$$
\operatorname{deg} \mathcal{L}=\left(2 g-2+n-\sum m_{i}\right) / r
$$

therefore $\chi(C, \mathcal{L})=1-g+\left(2 g-2+n-\sum m_{i}\right) / r$. We denote this integer by $\chi_{r, \mathbf{m}}$.

1.2. Principal $\mathbb{G}_{\mathbf{m}}$-bundles and $r$-spin curves. We now introduce another category which is equivalent to $\mathcal{M}_{g, n}^{1 / r, \mathbf{k}}$ when all $k_{i}$ are -1 .

First consider $\mathcal{B} \mathbb{G}_{\mathbf{m}}$, the classifying stack of $\mathbb{G}_{\mathbf{m}}$. This is an Artin algebraic stack whose objects over a scheme $S$ are principal $\mathbb{G}_{\mathbf{m}}$-bundles $P \rightarrow S$ and whose morphisms are fiber diagrams. Recall that such a bundle is always affine. Indeed we have

$$
P=\operatorname{Spec}_{\mathcal{O}_{S}}\left(\bigoplus_{i \in \mathbb{Z}} \mathcal{L}^{i}\right),
$$

where $\mathcal{L}$ is an invertible sheaf on $S$. The total space of $\mathcal{L}$ is $\operatorname{Spec}_{\mathcal{O}_{S}}\left(\bigoplus_{i \geq 0} \mathcal{L}^{i}\right)$. Thus, as usual, the data of $P \rightarrow S$ and $\mathcal{L}$ are interchangeable.

The $r$ th power map $\mathbb{G}_{\mathbf{m}} \rightarrow \mathbb{G}_{\mathbf{m}}$ induces a 1 -morphism $\kappa_{r}: \mathcal{B} \mathbb{G}_{\mathbf{m}} \rightarrow \mathcal{B} \mathbb{G}_{\mathbf{m}}$ which sends $P \rightarrow S$ to $P / \boldsymbol{\mu}_{r} \rightarrow S$, or equivalently $\mathcal{L}$ to $\mathcal{L}^{r}$. Here

$$
P / \boldsymbol{\mu}_{r}=\operatorname{Spec}_{\mathcal{O}_{S}}\left(\bigoplus_{i \in r \mathbb{Z}} \mathcal{L}^{i}\right) .
$$

Let

$$
\left(C \rightarrow S, s_{i}: S \rightarrow C, \mathcal{L}, c: \mathcal{L}^{r} \rightarrow \omega_{C / S}\left(\sum S_{i}\right)\right)
$$

be an object of the open moduli stack $\mathcal{M}_{g, n}^{1 / r, \mathbf{k}}$ with all $k_{i}=-1$. Then we have a 1-commutative diagram

$$
C^{\mathcal{L} \nearrow} \stackrel{\mathcal{B} \mathbb{G}_{\mathbf{m}}}{\underset{\omega_{C / S}\left(\sum S_{i}\right)}{\longrightarrow}} \searrow \kappa_{r} \quad \mathcal{B} \mathbb{G}_{\mathbf{m}},
$$

where the isomorphism $\kappa_{r} \circ \mathcal{L} \rightarrow \omega_{C / S}\left(\sum S_{i}\right)$ is given by $c$. Indeed, such a diagram is equivalent to the data of an object of $\mathcal{M}_{g, n}^{1 / r, \mathbf{k}}$ with all $k_{i}=-1$. Thus the category $\mathcal{M}_{g, n}^{1 / r, \mathbf{k}}$ with all $k_{i}=-1$ can be defined as a category whose objects are diagrams as above, with morphism given by fiber diagrams.

1.3. Twisted curves. To generalize the observation above for general $\mathbf{m}$, and over nodal curves, we use the notion of twisted curves of [1]. Recall that a twisted, $n$ pointed, nodal curve $\left(\mathcal{C} \rightarrow S, \mathcal{S}_{i}^{\mathcal{C}}\right)$ is a Deligne-Mumford stack $\mathcal{C}$, flat of relative dimension 1 over $S$, and $n$ closed substacks $\mathcal{S}_{i}^{\mathcal{C}} \subset \mathcal{C}$, such that $\mathcal{C}$ is nodal, $\mathcal{S}_{i}^{\mathcal{C}} \rightarrow S$ are étale gerbes, the coarse moduli space $\left(C, S_{i}\right)$ forms a proper, $n$-pointed, nodal curve over $S$, and $\pi: \mathcal{C} \rightarrow C$ is an isomorphism away from the nodes and the markings $S_{i}$.

Twisted curves over a strictly Henselian local ring $R$ have the following local structure: let $p$ be a geometric point of $\mathcal{C}$. Denote by $\mathcal{C}^{\text {sh }}$ the strict Henselization of $\mathcal{C}$ at $p$.

- If $p$ does not lie over a node or a marking, then $\mathcal{C}$ is isomorphic to $C$ around $p$, and therefore $\mathcal{C}^{\text {sh }} \simeq \operatorname{Spec} R[x]^{\text {sh }}$. 
- If $p$ lies over a marking $\mathcal{S}_{i}$, then there is an integer $l$ and an isomorphism $\mathcal{C}^{\text {sh }} \simeq\left[U / \boldsymbol{\mu}_{l}\right]$, where $U=\operatorname{Spec} R[z]^{\text {sh }}$ and $\boldsymbol{\mu}_{l}$ acts on $z$ via the standard representation $z \mapsto \zeta_{l} z$. We also have $C^{\text {sh }} \simeq \operatorname{Spec} R[x]^{\text {sh }}$, where $x=z^{l}$.

At such a point, the dualizing sheaf corresponds to the $\boldsymbol{\mu}_{l}$-equivariant sheaf $\omega_{U}$, with generator $d z$, on which $\boldsymbol{\mu}_{l}$ acts via the standard character. It follows that $d z / z=l^{-1}(d x / x)$ is an invariant generator of $\omega_{\mathcal{C}}\left(\mathcal{S}_{i}\right)$.

- If $p$ lies over a node, there is again an integer $l$ and an isomorphism $\mathcal{C}^{\text {sh }} \simeq$ $\left[U / \boldsymbol{\mu}_{l}\right]$, where $U=\operatorname{Spec}(R[z, w] /(z w-t))^{\text {sh }}$ for some $t \in R$ and $\boldsymbol{\mu}_{l}$ acts on $(z, w)$ via $(z, w) \mapsto\left(\zeta_{l} z, \zeta_{l}^{a} w\right)$ for some $a \in(\mathbb{Z} / l \mathbb{Z})^{\times}$. We also have $C^{\text {sh }} \simeq$ $\operatorname{Spec}\left(R[x, y] /\left(x y-t^{l}\right)\right)^{\text {sh }}$, where $x=z^{l}$ and $y=w^{l}$.

At such a point, the dualizing sheaf corresponds to the $\boldsymbol{\mu}_{l}$-equivariant sheaf $\omega_{U}$, with invariant generator $\nu_{*}^{U}(d z / z-d w / w)=l^{-1} \nu_{*}^{C}(d x / x-d y / y)$, where $\nu$ stands for normalization. The sheaf of Kähler differentials corresponds to the $\boldsymbol{\mu}_{l}$-equivariant sheaf $\Omega_{U}^{1}$, with generators $d z, d w$ (with $\boldsymbol{\mu}_{l}$-action as above) and relation $w d z+z d w=0$.

This analysis implies that $\omega_{\mathcal{C} / S}\left(\sum \mathcal{S}_{i}^{\mathcal{C}}\right)$ is isomorphic to the pullback of $\omega_{C / S}\left(\sum S_{i}\right)$. For simplicity we denote $\omega_{\log }=\omega_{\log }^{\mathcal{C}}=\omega_{\mathcal{C} / S}\left(\sum \mathcal{S}_{i}^{\mathcal{C}}\right)$.

The twisted stable curve is balanced if the action of the stabilizer $G_{p} \simeq \boldsymbol{\mu}_{l}$ at a nodal geometric point $p$ of $\mathcal{C}$ on the tangent spaces of the two branches of $\mathcal{C}$ at $p$ has complementary eigenvalues, i.e., $a=l-1$.

1.4. Twisted $r$-spin curves. A twisted $r$-spin curve is a 1-commutative diagram

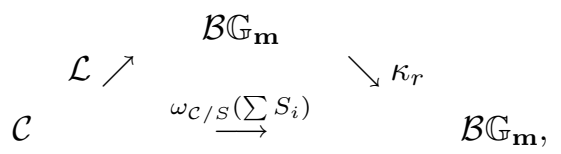

where

(1) $\left(\mathcal{C}, \mathcal{S}_{i}^{\mathcal{C}}\right)$ is a balanced twisted nodal curve,

(2) $\left(C, S_{i}\right)$ is a stable pointed curve, and

(3) the morphism (or line bundle) $\mathcal{L}: \mathcal{C} \rightarrow \mathcal{B} \mathbb{G}_{\mathbf{m}}$ is representable; in other words, the action of the local automorphism groups of $\mathcal{C}$ on the fibers of the line bundle $\mathcal{L}$ are faithful.

Morphisms of twisted $r$-spin curves are given by 1-commutative fiber diagrams, up to 2-isomorphisms, as in [1, Proposition 4.2.2]. We denote this category by $\mathcal{B}_{g, n}\left(\mathbb{G}_{\mathbf{m}}, \omega_{\log }^{1 / r}\right)$.

1.5. Twisted $r$-spin curves and twisted stable maps. We now identify $\mathcal{B}_{g, n}\left(\mathbb{G}_{\mathbf{m}}, \omega_{\log }^{1 / r}\right)$ with a certain stack of twisted stable maps. Let $C_{g, n} \rightarrow \overline{\mathcal{M}}_{g, n}$ be the universal curve. Consider the stack

$$
C_{g, n}\left(\omega_{\log }^{1 / r}\right)=C_{g, n} \underset{\mathcal{B G}_{\mathbf{m}}}{\times} \mathcal{B} \mathbb{G}_{\mathbf{m}}
$$

where the fibered product is taken with respect to the morphism

$$
C \stackrel{\omega_{\log }}{\longrightarrow} \mathcal{B} \mathbb{G}_{\mathbf{m}}
$$

on the left and with respect to $\kappa_{r}: \mathcal{B} \mathbb{G}_{\mathbf{m}} \rightarrow \mathcal{B} \mathbb{G}_{\mathbf{m}}$ on the right.

The stack $C_{g, n}\left(\omega_{\log }^{1 / r}\right)$ is the stack of $r$ th roots of $\omega_{C_{g, n} / \overline{\mathcal{M}}_{g, n}}\left(\sum S_{i}\right)$ over $C_{g, n}$, which is an étale gerbe over $C_{g, n}$ banded by $\boldsymbol{\mu}_{r}$. In particular it is a DeligneMumford stack. 
A twisted $r$-spin curve gives rise to a representable map $\mathcal{C} \rightarrow C_{g, n}\left(\omega_{\log }^{1 / r}\right)$, which is a balanced twisted stable map. The homology class of the image of the coarse curve $C$ is the class $F$ of a fiber of the universal curve $C_{g, n} \rightarrow \overline{\mathcal{M}}_{g, n}$ : the family of coarse curves $C \rightarrow S$ gives rise to a moduli morphism $S \rightarrow \overline{\mathcal{M}}_{g, n}$ and we have an isomorphism $C \simeq S \times \overline{\mathcal{M}}_{g, n} C_{g, n}$. We thus have a base-preserving functor from $\mathcal{B}_{g, n}\left(\mathbb{G}_{\mathbf{m}}, \omega_{\log }^{1 / r}\right)$ to the stack $\mathcal{K}_{g, n}^{b a l}\left(C_{g, n}\left(\omega_{\log }^{1 / r}\right) / \overline{\mathcal{M}}_{g, n}, F\right)$, the stack of balanced, $n$ pointed twisted stable maps of genus $g$ and class $F$ into $C_{g, n}\left(\omega_{\log }^{1 / r}\right)$ relative to the base stack $\overline{\mathcal{M}}_{g, n}$ (see [1], esp. Section 8.3). The image lies in the closed substack where the markings of $C$ line up over the markings of $C_{g, n}$ (i.e. the inverse image of $\prod_{i=1}^{n} S_{i}^{C_{g, n}}$ under the evaluation map to $C_{g, n}^{n}$ ). It is easy to see that the resulting functor is an equivalence. We thus have:

Theorem 1.5.1. The category $\mathcal{B}_{g, n}\left(\mathbb{G}_{\mathbf{m}}, \omega_{\log }^{1 / r}\right)$ is equivalent to the closed substack of $\mathcal{K}_{g, n}^{b a l}\left(C_{g, n}\left(\omega_{\log }^{1 / r}\right) / \overline{\mathcal{M}}_{g, n}, F\right)$, where the inverse image of $S_{i}^{C_{g, n}}$ is $\mathcal{S}_{i}^{C}$. In particular, it is a Deligne-Mumford stack admitting a projective coarse moduli space.

\section{INFINITESIMAL STRUCTURE OF THE STACK OF TWISTED SPIN CURVES}

\subsection{Obstructions.}

Proposition 2.1.1. The stack $\mathcal{B}_{g, n}\left(\mathbb{G}_{\mathbf{m}}, \omega_{\log }^{1 / r}\right)$ is smooth.

Proof. The relative cotangent complex $\mathbb{L}_{\kappa_{r}}$ of $\kappa_{r}: \mathcal{B} \mathbb{G}_{\mathbf{m}} \rightarrow \mathcal{B} \mathbb{G}_{\mathbf{m}}$ is trivial, therefore deformations and obstructions of a twisted spin curve are identical to those of the underlying pointed twisted curves.

As in [1. Lemma 5.3.3], obstructions of a pointed twisted curve $\left(\mathcal{C} \rightarrow S, \mathcal{S}_{i}^{\mathcal{C}}\right)$ are the same as obstructions of $\mathcal{C} \rightarrow S$, given by $\operatorname{Ext}^{2}\left(\Omega_{\mathcal{C}}^{1}, \mathcal{O}_{\mathcal{C}}\right)$. As in $[3]$, in degree 2 the local-to-global spectral sequence for Ext involves the terms $H^{2}\left(\mathcal{C}, \mathcal{H o m}\left(\Omega_{\mathcal{C}}^{1}, \mathcal{O}_{\mathcal{C}}\right)\right.$ ), $H^{1}\left(\mathcal{C}, \mathcal{E} x t^{1}\left(\Omega_{\mathcal{C}}^{1}, \mathcal{O}_{\mathcal{C}}\right)\right)$, and $H^{0}\left(\mathcal{C}, \mathcal{E} x t^{2}\left(\Omega_{\mathcal{C}}^{1}, \mathcal{O}_{\mathcal{C}}\right)\right)$. The first vanishes because of dimension reasons. Similarly, $\mathcal{E} x t^{1}\left(\Omega_{\mathcal{C}}^{1}, \mathcal{O}_{\mathcal{C}}\right)$ is supported in dimension 0 ; hence the second term vanishes. Thus only the third term remains. We claim that the sheaf $\mathcal{E} x t^{2}\left(\Omega_{\mathcal{C}}^{1}, \mathcal{O}_{\mathcal{C}}\right)$ vanishes. This follows since locally $\Omega_{\mathcal{C}}^{1}$ has a 2 -term locally free resolution. For instance, at a node where $\mathcal{C} \simeq\left[U / \boldsymbol{\mu}_{l}\right]$ with $U=\operatorname{Spec}(k[z, w] /(z w))^{\mathrm{sh}}$, we have a $\boldsymbol{\mu}_{l}$-equivariant exact sequence on $U$ :

$$
0 \rightarrow \mathcal{O}_{U} \stackrel{(z, w)}{\longrightarrow} \mathcal{O}_{U} \oplus \mathcal{O}_{U} \stackrel{(d w+d z)}{\longrightarrow} \Omega_{U}^{1} \rightarrow 0
$$

with appropriate $\boldsymbol{\mu}_{l}$-weights, giving a locally free resolution of $\Omega_{\mathcal{C}}^{1}$. Thus twisted curves are unobstructed.

2.2. Deformations. To determine the dimension of the stack of twisted spin curves, it suffices to identify the deformation space of a twisted pointed curve.

Proposition 2.2.1. The tangent space of the stack of twisted curves at a point $\left(\mathcal{C}, \mathcal{S}_{i}^{\mathcal{C}}\right)$, such that the coarse pointed curve is stable, has dimension $3 g-3+n-u$, where $u$ is the number of nodes in $\mathcal{C}$ which are not balanced.

Proof. As in 1, Lemma 5.3.2], we have an exact sequence

$$
\operatorname{Hom}\left(\Omega_{\mathcal{C}}^{1}, \mathcal{O}_{\mathcal{C}}\right) \rightarrow H^{0}\left(\mathcal{C}, \oplus \mathcal{N}_{\mathcal{S}_{i}^{\mathcal{C}}}\right) \rightarrow \operatorname{Def} \rightarrow \operatorname{Ext}^{1}\left(\Omega_{\mathcal{C}}^{1}, \mathcal{O}_{\mathcal{C}}\right) \rightarrow 0
$$


where Def is the tangent space of the stack. The kernel of the map on the left is the space of infinitesimal automorphisms of $\left(\mathcal{C}, \mathcal{S}_{i}^{\mathcal{C}}\right)$, which is trivial by the stability assumption.

At points where $\mathcal{S}_{i}$ is untwisted, we have that $H^{0}\left(\mathcal{C}, \mathcal{N}_{\mathcal{S}_{i}^{c}}\right)$ has dimension 1. But at twisted markings the normal space $\mathcal{N}_{\mathcal{S}_{i}^{c}}$ has no non-trivial local sections: locally we have an isomorphism $\left[U / \boldsymbol{\mu}_{l}\right] \rightarrow \mathcal{C}^{\text {sh }}$, where $U=\operatorname{Spec}(k[z])^{\text {sh }}$ with the standard action of $\boldsymbol{\mu}_{l}$; therefore, $\boldsymbol{\mu}_{l}$ acts on a generator $\partial / \partial z$ of $\mathcal{N}_{\mathcal{S}_{i}^{c}}$ via the non-trivial character $\zeta_{l} \mapsto \zeta_{l}^{-1}$.

The group $\operatorname{Ext}^{i}\left(\Omega_{\mathcal{C}}^{1}, \mathcal{O}_{\mathcal{C}}\right)$ is dual to $H^{1-i}\left(\mathcal{C}, \Omega_{\mathcal{C}}^{1} \otimes \omega_{\mathcal{C}}\right)$. By [1, Lemma 2.3.4], this is the same as $H^{1-i}\left(C, \pi_{*}\left(\Omega_{\mathcal{C}}^{1} \otimes \omega_{\mathcal{C}}\right)\right)$, where $\pi: \mathcal{C} \rightarrow C$ is the natural map. Let us compare $\pi_{*}\left(\Omega_{\mathcal{C}}^{1} \otimes \omega_{\mathcal{C}}\right)$ with $\Omega_{C}^{1} \otimes \omega_{C}$. These are clearly isomorphic away from the twisted markings and the twisted nodes.

First consider a twisted marking $\mathcal{S}_{i}$ where we have an isomorphism $\left[U / \boldsymbol{\mu}_{l}\right] \rightarrow \mathcal{C}^{\text {sh }}$ with $U$ as above. The action of $\boldsymbol{\mu}_{l}$ on $d z$ is via the standard character, therefore the invariant quadratic differentials are generated by $z^{l}(d z / z)^{2}=l^{-2} x(d x / x)^{2}=$ $l^{-2}(d x)^{2} / x$, where $x$ is a parameter on $C$. That is, locally near such a marking we have $\pi_{*}\left(\Omega_{\mathcal{C}}^{1} \otimes \omega_{\mathcal{C}}\right)=\Omega_{C}^{1} \otimes \omega_{C}\left(S_{i}\right)$.

Now consider a node on $\mathcal{C}$ with an isomorphism $\left[U / \boldsymbol{\mu}_{l}\right] \rightarrow \mathcal{C}^{\text {sh }}$ with $U=$ $\operatorname{Spec}(k[z, w] /(z w))^{\mathrm{sh}}$, and the action can be described via $(z, w) \mapsto\left(\zeta_{l} z, \zeta_{l}^{a} w\right)$ for some $a \in(\mathbb{Z} / l \mathbb{Z})^{\times}$. The sheaf $\omega_{\mathcal{C}}$ has an invariant generator $\nu_{*}(d z / z-d w / w)$, where $\nu$ is the normalization. The sheaf $\Omega_{\mathcal{C}}^{1}$ has sections $f(z) d z+g(w) d w+\alpha z d w$. Invariant elements of the form $f(z) d z+g(w) d w$ are exactly $\Omega_{C}^{1} /$ torsion, whereas $z d w$ is invariant if and only if $a=-1$, i.e., the node is balanced!

All in all we have $\pi_{*}\left(\Omega_{\mathcal{C}}^{1} \otimes \omega_{\mathcal{C}}\right)=\Omega_{C}^{1} \otimes \omega_{C}\left(\sum S_{j}\right) / \mathcal{T}$, where the sum $\sum S_{j}$ is taken over the twisted markings, and the sheaf $\mathcal{T}$ is the torsion subsheaf supported at unbalanced nodes. The Euler characteristic agrees with that of $\left(C, S_{i}\right)$ with the exception of the torsion sections at these unbalanced nodes. The proposition follows.

Corollary 2.2.2. The morphism $\mathcal{B}_{g, n}\left(\mathbb{G}_{\mathbf{m}}, \omega_{\log }^{1 / r}\right) \rightarrow \overline{\mathcal{M}}_{g, n}$ is flat, proper and quasifinite.

\section{Twisted spin Curves And Admissible $\mathbb{G}_{\mathbf{m}}$-Bundles}

Let $\left(\mathcal{C} \rightarrow S, \mathcal{S}_{i}^{\mathcal{C}}, \mathcal{L}, c\right)$ be a twisted stable $r$-spin curve. We have a corresponding principal $\mathbb{G}_{\mathbf{m}}$-bundle $P_{\mathcal{L}} \stackrel{p}{\rightarrow} \mathcal{C}$. Recall that the universal principal bundle $\mathcal{P} \mathbb{G}_{\mathbf{m}} \rightarrow$ $\mathcal{B} \mathbb{G}_{\mathbf{m}}$ is representable - in fact, $\mathcal{P} \mathbb{G}_{\mathbf{m}}$ is the base scheme $S$. Since $\mathcal{L}$ is representable, we have that $P_{\mathcal{L}}$ is representable by an algebraic space. We denote by $\bar{p}: P_{\mathcal{L}} \rightarrow C$ the composition $\pi \circ p$, where again $\pi: \mathcal{C} \rightarrow C$ is the natural map from $\mathcal{C}$ to its coarse moduli $C$.

As indicated before, the map $p: P_{\mathcal{L}} \rightarrow \mathcal{C}$ is affine. Since the functor $\pi_{*}:$ $\operatorname{Mod}_{\mathcal{O}_{\mathcal{C}}} \rightarrow \operatorname{Mod}_{\mathcal{O}_{C}}$ is exact, we have that $\bar{p}_{*}$ is exact on quasi-coherent sheaves, which means, by Serre's criterion, that $\bar{p}: P_{\mathcal{L}} \rightarrow C$ is affine. Explicitly, we have $P_{\mathcal{L}}=\operatorname{Spec}_{\mathcal{O}_{\mathcal{C}}}\left(\bigoplus_{i \in \mathbb{Z}} \mathcal{L}^{i}\right)$, and denoting $\mathcal{E}_{i}=\pi_{*} \mathcal{L}^{i}$, we have

$$
P_{\mathcal{L}}=\operatorname{Spec}_{\mathcal{O}_{C}}\left(\bigoplus_{i \in \mathbb{Z}} \mathcal{E}_{i}\right)
$$


The $\mathbb{Z}$-grading reflects the fact that we still have a $\mathbb{G}_{\mathbf{m}}$-action, making $P_{\mathcal{L}}$ a homogeneous $\mathbb{G}_{\mathbf{m}}$-fibration. Of course $P_{\mathcal{L}} \rightarrow C$ is not necessarily a principal bundle unless $\pi$ is an isomorphism. Let us make the structure of $P_{\mathcal{L}}$ more explicit.

Recall that $\mathcal{L}^{r} \simeq \omega_{\log }$. This means that $\left[P_{\mathcal{L}} / \boldsymbol{\mu}_{r}\right]=P_{\omega_{\text {log }}}$ is the principal bundle associated to $\omega_{\log }$. On the other hand, the schematic quotient $P_{\mathcal{L}} / \boldsymbol{\mu}_{r}=$ $\operatorname{Spec}_{\mathcal{O}_{C}}\left(\bigoplus_{i \in r \mathbb{Z}} \mathcal{E}_{i}\right)$. But $\mathcal{E}_{r k}=\pi_{*} \mathcal{L}^{r k}=\left(\pi_{*} \omega_{\log }^{\mathcal{C}}\right)^{k}=\left(\omega_{\log }^{C}\right)^{k}$. Thus $P_{\mathcal{L}} / \boldsymbol{\mu}_{r} \simeq P_{\omega_{\text {log }}^{C}} \rightarrow$ $C$ is the principal bundle associated to $\omega_{\log }^{C}$.

We can describe $P_{\mathcal{L}}$ over twisted markings and nodes as follows: if $\mathcal{C}^{\text {sh }} \simeq\left[U / \boldsymbol{\mu}_{l}\right]$, then the pullback of $P_{\mathcal{L}}$ to $U$ is isomorphic to $U \times \mathbb{G}_{\mathbf{m}}$ where $\boldsymbol{\mu}_{l}$ acts on $\mathbb{G}_{\mathbf{m}}$ via some non-trivial character. It follows that $\left.P_{\mathcal{L}}\right|_{C^{\mathrm{sh}}} \simeq U \times \mathbb{G}_{\mathbf{m}} / \boldsymbol{\mu}_{l}$. At a twisted marking this implies that $P_{\mathcal{L}}$ is non-singular, with fiber of multiplicity $l$. Over a node, $P_{\mathcal{L}}$ is a normal crossings surface whose double curve is the reduction of the fiber. Moreover, the fact that $\mathcal{C}$ is balanced implies that $\left.P_{\mathcal{L}}\right|_{C^{\mathrm{sh}}}$ has the form $\operatorname{Spec} R\left[z, w, \xi, \xi^{-1}\right] /(z w-t)^{\mathrm{sh}} / \boldsymbol{\mu}_{l}$ where the action of $\boldsymbol{\mu}_{l}$ on $z$ and $w$ has characters inverse to each other. We call such $P$ balanced.

We can now reverse this process: define a category $\mathcal{P}_{g, n}\left(\omega_{\log }^{1 / r}\right)$ whose objects are $\left(P \rightarrow C \rightarrow S, S_{i}, c\right)$, where

(1) $\left(C \rightarrow S, S_{i}\right)$ is a stable $n$-pointed curve of genus $g$,

(2) $P \rightarrow S$ is a flat family of normal crossing surfaces,

(3) $P \rightarrow C$ is a balanced, homogeneous $\mathbb{G}_{\mathbf{m}}$-fibration, which is principal on the complement of the nodes and markings, and

(4) $c: P / \boldsymbol{\mu}_{r} \rightarrow P_{\omega_{\log }^{C}}$ an isomorphism, which is $\mathbb{G}_{\mathbf{m}}$-equivariant when the action of $\mathbb{G}_{\mathbf{m}}$ on $P_{\omega_{\text {log }}^{C}}$ is defined via the $r$ th-power map $\mathbb{G}_{\mathbf{m}} \rightarrow \mathbb{G}_{\mathbf{m}}$.

Morphisms are defined using fiber diagrams as usual.

We have:

Proposition 3.0.1. The stacks $\mathcal{P}_{g, n}\left(\omega_{\log }^{1 / r}\right)$ and $\mathcal{B}_{g, n}\left(\mathbb{G}_{\mathbf{m}}, \omega_{\log }^{1 / r}\right)$ are isomorphic.

Proof. The argument above gives a functor $\mathcal{B}_{g, n}\left(\mathbb{G}_{\mathbf{m}}, \omega_{\log }^{1 / r}\right) \rightarrow \mathcal{P}_{g, n}\left(\omega_{\log }^{1 / r}\right)$. In the reverse direction, given $\left(P \rightarrow C \rightarrow S, S_{i}, c\right)$, consider the stack $\mathcal{C}=\left[P / \mathbb{G}_{\mathbf{m}}\right]$. By condition (4), $P$ has finite stabilizers inside the reduced group scheme $\boldsymbol{\mu}_{r}$, therefore $\mathcal{C}$ is a Deligne-Mumford stack. Clearly $P \rightarrow \mathcal{C}$ is a principal bundle. Also the associated morphism $\mathcal{C} \rightarrow \mathcal{B} \mathbb{G}_{\mathbf{m}}$ is representable since $P$ is. The local structure of $P$ implies that $\mathcal{C}$ is nodal and balanced.

It is easy to see that the two compositions of these functors are equivalent to the identity.

Let us study the algebra $\bigoplus_{i \in \mathbb{Z}} \mathcal{E}_{i}$ and its constituents locally in more detail. On the open set where $\pi$ is an isomorphism, we have $\mathcal{E}_{i}=\mathcal{L}^{i}$, which is invertible, and $\mathcal{E}_{1}=\mathcal{L}$ is an $r$ th root of $\omega_{\log }$.

Consider an étale neighborhood of a geometric point over a twisted marking $\mathcal{S}_{i}^{\mathcal{C}}$, where $\mathcal{C}^{\text {sh }} \simeq\left[U / \boldsymbol{\mu}_{l}\right]$ and $U=\operatorname{Spec}(R[z])^{\text {sh }}$. The sheaf $\mathcal{L}$ corresponds to an equivariant invertible sheaf on $U$, also denoted $\mathcal{L}$, such that $\mathcal{L}^{r} \simeq \omega_{\log }$ is invariant. Choose a semi-invariant generator $s$ of $\mathcal{L}$ such that $s^{r}=d z / z$. The group $\boldsymbol{\mu}_{l}$ acts on $s$ via a character $\rho_{b}$ given by $\zeta_{l} \mapsto \zeta_{l}^{b}$. This implies in particular that $s^{i}$ is a generator of $\mathcal{L}^{i}$ with character $\rho_{b}^{i}$. The representability assumption means that $b \in(\mathbb{Z} / l \mathbb{Z})^{\times}$.

A monomial section $z^{a} s$ of $\mathcal{L}$ is invariant if and only if $a+b \equiv 0 \bmod l$, therefore an invariant section has the form $f\left(z^{l}\right) z^{a} s=f(x) z^{a} s$, where $a=l-b$. In particular, 
$\pi_{*} \mathcal{L}$ is an invertible sheaf on $U / \boldsymbol{\mu}_{l}$ generated by $z^{a} s$. And $\left(\pi_{*} \mathcal{L}\right)^{r}$ is generated by $\left(z^{a} s\right)^{r}=x^{a r / l}(d z / z)=l^{-1} x^{a r / l}(d x / x)$. If we let $k=a r / l$, then we have that $\pi_{*} \mathcal{L}$ is an $r$ th root of $\omega_{\log }(-k S)$ on $U / \boldsymbol{\mu}_{l}$.

A similar analysis holds for $\mathcal{E}_{i}$. A section $z^{a} s^{i}$ is invariant if $a+i b \equiv 0 \bmod l$. Denote by $k_{i}$ the least non-negative integer such that $k_{i} \equiv-i b(r / l) \bmod r$. Then $\mathcal{E}_{i}$ is an $r$ th root of $\omega_{\log }^{i}\left(-k_{i} S\right)$ on $U / \boldsymbol{\mu}_{l}$.

Consider now an étale neighborhood of a geometric point over a twisted node. Here $\mathcal{C}^{\text {sh }} \simeq\left[U / \boldsymbol{\mu}_{l}\right]$ and $U=\operatorname{Spec}(R[z, w] /(z w-t))^{\text {sh }}$. Recall that, according to our notation, $\boldsymbol{\mu}_{l}$ acts on $z$ via the standard character $\rho_{1}$ and on $w$ via its inverse $\rho_{l-1}$. Again we choose a semi-invariant generator $s$ of $\mathcal{L}$, with character $\rho_{b}$, such that $s^{r}=\nu_{*}(d z / z-d w / w)$, where $\nu$ denotes normalization. Again the representability assumption implies that $b \in \mathbb{Z} / l \mathbb{Z}^{\times}$. A monomial section $z^{i} s$ is invariant if $i+b \equiv 0$ $\bmod l$, and a monomial section $w^{j} s$ is invariant if $-j+b \equiv 0 \bmod l$. Thus if $a=$ $l-b$, the sheaf $\mathcal{E}_{1}$ is the torsion free sheaf generated by $u=z^{a} s$ and $v=w^{b} s$, where $y u=t^{a} v$ and $x v=t^{b} u$. Moreover, $u^{r}=l^{-1} x^{a r / l}(d x / x)$ and $v^{r}=l^{-1} y^{b r / l}(d y / y)$. In the notation of Section 4.2, below, (or of [5]) the sheaf $\mathcal{E}_{1}$ is locally isomorphic to $E_{a, b}$.

Again, a similar analysis holds for $\mathcal{E}_{i}$.

\section{Comparison of TWISTED SPIN CURVES AND QUASI-SPIN CURVES}

We recall some definitions from [4] and [5]

4.1. Moduli of stable $r$-quasi-spin curves. In [4], a natural compactification of $\mathcal{M}_{g, n}^{1 / r, \mathbf{k}}$ using torsion free sheaves is proposed. The crucial point is that as a smooth $r$-spin curve degenerates to a nodal curve $C_{0}$, the sheaf $\mathcal{L}$ must often degenerate to a torsion free sheaf at the nodes of $C_{0}$. But, as shown in [4], only special types of degeneration occur.

An $n$-pointed stable $r$-quasi-spin curve of genus $g$ and type $\mathbf{m}-\mathbf{1}$ over a scheme $S$ is the data of

(1) a stable $n$-pointed curve $\left(C \rightarrow S, s_{i}: S \rightarrow C\right)$ of genus $g$,

(2) a torsion free sheaf $\mathcal{F}$ on $C$ with $\chi(C, \mathcal{F})=\chi_{r, \mathbf{m}}$, and

(3) a morphism $\mathcal{F}^{\otimes r} \rightarrow \omega_{C / S}\left(\sum_{i=1}^{n}\left(1-m_{i}\right) S_{i}\right)$, which restricts to an isomorphism on the open set where $\mathcal{F}$ is locally free, and such that the length of the cokernel at each node where $\mathcal{F}$ is not locally free is precisely $r-1$.

The last condition on the cokernel of $c$ is comparable to the fact for twisted spin curves that the local index near each node divides $r$.

Morphisms of stable $r$-quasi-spin curves of genus $g$ and type $\mathbf{m}-\mathbf{1}$ are defined using fiber diagrams as above. The category $\overline{\mathcal{Q}}_{g, n}^{1 / r, \mathbf{m}-1}$ of $n$-pointed, stable, $r$-quasispin curve of genus $g$ and type $\mathbf{m} \mathbf{- 1}$ with morphisms given by fiber diagrams is a proper Deligne-Mumford stack with projective coarse moduli space $\overline{\mathbf{Q}}_{g, n}^{1 / r, \mathbf{m}-\mathbf{1}}$. For proofs see [4].

4.2. Moduli of stable $r$-spin curves. The stack $\overline{\mathcal{Q}}_{g, n}^{1 / r, \mathbf{k}} \rightarrow \operatorname{Spec} \mathbb{Z}[1 / r]$ is not smooth. To correct this, a refined structure on nodal curves is introduced in [5]. This structure involves restrictions on the way the spin structures may vary in families and it involves additional data in the form of intermediate roots of $\omega_{\text {log }}$.

As mentioned in Section 1.1 the usual definition [5] of an $r$-spin curve of type $\mathbf{m}$ is given in terms of roots of $\omega$ instead of $\omega_{\log }$. The shift from $\omega$ to $\omega_{\log }$ adjusts the 
type from $\mathbf{m}$ to $\mathbf{m}-\mathbf{1}$, but even accounting for this difference, the two definitions are not exactly the same. Still, as explained in [5. Note 2.1], although these two definitions are not identical, there is a canonical isomorphism between the stacks. Thus we will use the notation $\overline{\mathcal{M}}_{g, n}^{1 / r, \mathbf{m}-\mathbf{1}}$ to denote what we are calling $r$-spin curves of type $\mathbf{m}-\mathbf{1}$, despite the slight difference between this definition and that given in 5 .

An $n$-pointed stable $r$-spin curve of genus $g$ and type $\mathbf{m}-\mathbf{1}$ over a scheme $S$ is the data of

(1) a stable $n$-pointed curve $\left(C \rightarrow S, s_{i}: S \rightarrow C\right)$ of genus $g$,

(2) a torsion free sheaf $\mathcal{F}_{d}$ on $C$ for each $d \mid r$,

(3) an isomorphism $\mathcal{F}_{1} \stackrel{\sim}{\longrightarrow} \omega_{C / S}\left(\sum_{i=1}^{n} S_{i}\right)$, and

(4) for each pair $(d, e)$ of positive integers with $d|e| r$, a morphism $c_{e, d}: \mathcal{F}_{e}^{\otimes e / d} \rightarrow$ $\mathcal{F}_{d}$ which restricts to an isomorphism away from the nodes and marked points of $C$,

such that the following hold:

(1) For each $d \mid r$ let $m_{i}^{d}$ be the unique non-negative integer less than $d$ such that $m_{i}^{d} \equiv m_{i} \bmod d$. Write $\mathbf{m}^{d}=\left(m_{1}^{d}, \ldots, m_{n}^{d}\right)$. Then $c_{d, 1}: \mathcal{F}_{d}^{\otimes d} \rightarrow \mathcal{F}_{1}$ factors through $\mathcal{F}_{1}\left(-\sum m_{i}^{d} S_{i}\right) \simeq \omega_{C / S}\left(\sum\left(1-m_{i}^{d}\right) S_{i}\right)$, making $\left(C, s_{i}, \mathcal{F}_{d}, c_{d, 1}\right)$ into a $d$-quasi spin curve of type $\mathbf{m}^{d}-\mathbf{1}$.

(2) For each $d \mid r$ the morphism $c_{d, d}$ is the identity.

(3) For each $d\left|d^{\prime}\right| d^{\prime \prime} \mid r$, we have $c_{d^{\prime}, d} \circ c_{d^{\prime \prime}, d^{\prime}}^{\otimes d^{\prime}}=c_{d^{\prime \prime}, d}$.

(4) For each $d|e| r$ the length of the cokernel of $c_{e, d}$ at each point where $\mathcal{F}_{e}$ is not locally free is precisely $(e / d)-1$.

Finally, we place three conditions on the local structure of these sheaves and homomorphisms at nodes where the sheaf $\mathcal{F}_{r}$ is not locally free:

(1) First, when $\mathcal{F}_{r}$ is not locally free at a node $\mathfrak{p}$ in a fiber $C_{s}$ of $C / S$ over a geometric point $s \in S$, there must be an isomorphism of the strict Henselization $\mathcal{O}_{C, \mathfrak{p}}^{\text {sh }}$ of the local ring of $C$ at $\mathfrak{p}$ to the ring $A:=\left(R[x, y] / x y-t^{l}\right)^{\text {sh }}$, where $l$ is some integer dividing $r$, the ring $R=\mathcal{O}_{S, s}^{\text {sh }}$ is the strict Henselization of the base at $s$, and the element $t$ is in $R$.

(2) Second, for each pair of positive integers $i$ and $j$, such that $i+j=l$ we define the $A$-module $E_{i, j}:=\left\langle\xi_{1}, \xi_{2} \mid t^{j} \xi_{1}=x \xi_{2}, t^{i} \xi_{2}=y \xi_{1}\right\rangle$, and for notational convenience when $i=j=0$ we define $E_{0,0}$ to be the free $A$-module with the unusual presentation $E_{0,0}:=\left\langle\xi_{1}, \xi_{2} \mid \xi_{1}=\xi_{2}\right\rangle$, even if $t=0$. As explained in Sections 3 and 1.3. if $\mathcal{O}_{\mathcal{C}, \mathfrak{p}}^{\text {sh }} \cong \operatorname{Spec}(R[z, w] /(z w-t))^{\mathrm{sh}}$, then $E_{a, b}$ corresponds to the pushforward $\pi_{*} L$ of the free $\mathcal{O}_{\mathcal{C}, \mathfrak{p}}^{\text {sh }}$-module $L=\langle s\rangle$, where $\boldsymbol{\mu}_{l}$ acts on $s$ via the representation $\rho_{b}$. Given a choice of isomorphism $\mathcal{O}_{C, \mathfrak{p}}^{\text {sh }} \stackrel{\sim}{\rightarrow} A$, we require that for each $d$ dividing $r$, the strict Henselization $\mathcal{F}_{d}^{\text {sh }}$ at $\mathfrak{p}$ of each sheaf in the spin structure must be isomorphic to $E_{i_{d}, j_{d}}$, where $i_{d}$ and $j_{d}$ are the least non-negative integers congruent to $i_{r} d$ and $j_{r} d$ modulo $l$, respectively, and either $i_{r}+j_{r}=l$ or $i_{r}=j_{r}=0$.

Note that while the choice of $i_{r}$ and $j_{r}$ uniquely determines $i_{d}$ and $j_{d}$, it does not uniquely determine the isomorphism $\mathcal{F}_{d}^{\text {sh }} \stackrel{\sim}{\rightarrow} E_{i_{d}, j_{d}}$, since each $E_{i, j}$ has a non-trivial automorphism group.

(3) Finally, the homomorphisms $c_{d, e}: \mathcal{F}_{d}^{\otimes d / e} \rightarrow \mathcal{F}_{e}$ must form a compatible system of power maps [5, def. 2.3.1]. Essentially, this means that if $\mathcal{F}_{r}$ 
is the pushforward $\pi_{*} \mathcal{L}$ for some line bundle $\mathcal{L}$ on $\mathcal{C}$, then $\mathcal{F}_{d}$ is just the pushforward $\pi_{*} \mathcal{L}^{r / d}$ of the $r / d$ th power of $\mathcal{L}$, and $c_{e, d}$ corresponds to the map $\left(\pi_{*} \mathcal{L}^{r / e}\right)^{\otimes e / d} \rightarrow \pi_{*}\left(\mathcal{L}^{r / d}\right)$ induced by adjointness.

More specifically, this means that given $\mathcal{O}_{C, \mathfrak{p}}^{\text {sh }} \stackrel{\sim}{\rightarrow} A$ we require that for each $d$ dividing $r$ there is a choice of isomorphism $\mathcal{F}_{d}^{\text {sh }} \stackrel{\sim}{\rightarrow} E_{i_{d}, j_{d}}$, such that, in terms of the presentations $\mathcal{F}_{d}^{\text {sh }} \cong E_{i_{d}, j_{d}}=\left\langle\xi_{1}, \xi_{2} \mid t^{j_{d}} \xi_{1}=x \xi_{2}, y \xi_{1}=t^{i_{d}} \xi_{2}\right\rangle$, and $\mathcal{F}_{e}^{\text {sh }} \cong E_{i_{e}, j_{e}}=\left\langle\zeta_{1}, \zeta_{2} \mid t^{j_{e}} \zeta_{1}=x \zeta_{2}, y \zeta_{1}=t^{i_{e}} \zeta_{2}\right\rangle$, the induced map $\bar{c}_{d, e}$ : $\operatorname{Sym}^{d / e}\left(E_{i_{d}, j_{d}}\right) \rightarrow E_{i_{e}, j_{e}}$ can be written in terms of the generators $\xi_{1}^{(d / e)-k} \xi_{2}^{k}$ of $\mathbf{S y m}^{d / e}\left(E_{i_{d}, j_{d}}\right)$ as

$$
\xi_{1}^{(d / e)-k} \xi_{2}^{k} \mapsto \begin{cases}x^{u-k} t^{k j_{d}} \zeta_{1} & \text { if } 0 \leq k \leq u, \\ y^{v-d / e+k} t^{(d-k) i_{d}} \zeta_{2} & \text { if } u<k \leq d .\end{cases}
$$

Here we define $u:=\left((d / e) i_{d}-i_{e}\right) / l$ and $v:=\left((d / e) j_{d}-j_{e}\right) / l$.

In [5] it is shown that the property of being a compatible system of power maps is independent of the choice of $t \in R$ and of isomorphism $\mathcal{O}_{C, \mathfrak{p}}^{\text {sh }} \stackrel{\sim}{\rightarrow}\left(R[x, y] / x y-t^{l}\right)^{\mathrm{sh}}$. Moreover, the integers $i_{d}$ and $j_{d}$ are well defined. Of course, changing one of the isomorphisms $\mathcal{F}_{d}^{\text {sh }} \cong E_{i_{d}, j_{d}}$ will generally make the induced maps fail to be power maps; and indeed, given that these maps must be power maps, the choice of isomorphism $\mathcal{F}_{r}^{\text {sh }} \cong E_{i_{r}, j_{r}}$ uniquely determines all of the remaining isomorphisms for $d$ dividing $r$.

The category $\overline{\mathcal{M}}_{g, n}^{1 / r, \mathbf{m}-1}$ of $n$-pointed, stable $r$-spin curves of genus $g$ and type $\mathbf{m}-\mathbf{1}$, with morphisms given by fiber diagrams, is a proper Deligne-Mumford stack with projective coarse moduli space $\overline{\mathbf{M}}_{g, n}^{1 / r, \mathbf{m}-\mathbf{1}}$. It is shown in [5] that this stack is smooth over Spec $\mathbb{Z}[1 / r]$, for arbitrary $r$. The proof is quite subtle, as can be expected from the complexity of the definition.

There is a natural morphism $\overline{\mathcal{M}}_{g, n}^{1 / r, \mathbf{k}} \rightarrow \overline{\mathcal{Q}}_{g, n}^{1 / r, \mathbf{k}}$ obtained by forgetting $\mathcal{F}_{d}$ for $d \neq 1, r$. Since $\mathcal{F}_{d}$ is torsion free, any automorphism of this sheaf is determined by its value over the generic points of $C$, and therefore the morphism above is representable.

4.3. The functor $\mathcal{B}_{g, n}\left(\mathbb{G}_{\mathbf{m}}, \omega_{\log }^{1 / r}\right) \rightarrow \overline{\mathcal{M}}_{g, n}^{1 / r}$. Given a twisted stable $r$-spin curve $\left(\mathcal{C} \rightarrow S, \mathcal{S}_{i}, \mathcal{L}, c\right)$, for each positive integer $d$ dividing $r$, we have torsion free sheaves $\mathcal{F}_{r / d}=\pi_{*} \mathcal{L}^{d}$ on $C$, and the canonical isomorphisms $\left(\mathcal{L}^{d}\right)^{e / d} \rightarrow \mathcal{L}^{e}$ induce maps $c_{e, d}$ : $\mathcal{F}_{e}^{\otimes e / d} \rightarrow \mathcal{F}_{d}$. Consider the functor from $\mathcal{B}_{g, n}\left(\mathbb{G}_{\mathbf{m}}, \omega_{\log }^{1 / r}\right)$ to $\overline{\mathcal{M}}_{g, n}^{1 / r, \mathbf{k}}$ which associates to a twisted stable $r$-spin curve $\left(\mathcal{C} \rightarrow S, \mathcal{S}_{i}, \mathcal{L}, c\right)$ the data $\left(C \rightarrow S, S_{i},\left\{\mathcal{F}_{d}\right\},\left\{c_{e, d}\right\}\right)$. Based on the local analysis given in Section 3 it is easy to see that this functor lands in $\overline{\mathcal{M}}_{g, n}^{1 / r, \mathbf{k}}$ for some $\mathbf{k}$ determined by the local indices of the twisted spin curve. The general functor lands in the disjoint union

$$
\overline{\mathcal{M}}_{g, n}^{1 / r}:=\coprod_{-1 \leq k_{i} \leq r-2} \overline{\mathcal{M}}_{g, n}^{1 / r, \mathbf{k}} .
$$

By composing with the functor $\overline{\mathcal{M}}_{g, n}^{1 / r} \rightarrow \overline{\mathcal{Q}}_{g, n}^{1 / r}$ above, we also get a functor $\mathcal{B}_{g, n}\left(\mathbb{G}_{\mathbf{m}}, \omega_{\log }^{1 / r}\right) \rightarrow \mathcal{Q}_{g, n}^{1 / r}$

Note that given a balanced $\mathbb{G}_{\mathbf{m}}$-fibration $P \rightarrow C$, an automorphism $a \in$ $\operatorname{Aut}_{C}(P \rightarrow C)$ is determined by its action on the generic fibers of $P \rightarrow C$ over 
generic points of components of $C$. The action is thus determined by the induced action on the sheaf $\mathcal{E}_{1}$, or $\mathcal{F}_{r}$. It follows that both functors above are representable as well. Since they are quasi-finite and proper (these stacks being quasi-finite and proper over $\left.\overline{\mathcal{M}}_{g, n}\right)$ they are in fact finite. They are also birational, being compactifications of $\mathcal{M}_{g, n}^{1 / r, \mathbf{m}}$.

Recall that $\overline{\mathcal{M}}_{g, n}^{1 / r, \mathbf{m}}$ is smooth, in particular, normal. It follows that

Proposition 4.3.1. $\mathcal{B}_{g, n}\left(\mathbb{G}_{\mathbf{m}}, \omega_{\log }^{1 / r}\right) \rightarrow \overline{\mathcal{M}}_{g, n}^{1 / r}$ is an isomorphism.

4.4. Explicit realization of the inverse functor. We may see the inverse functor $\overline{\mathcal{M}}_{g, n}^{1 / r} \rightarrow \mathcal{P}_{g, n}\left(\omega_{l o g}^{1 / r}\right)$ directly, as follows. Given a stable $r$-spin curve $(C \rightarrow$ $S, S_{i},\left\{\mathcal{F}_{d}\right\},\left\{c_{d, d^{\prime}}\right\}$ ), we must define a $\mathbb{Z}$-graded $\mathcal{O}_{C^{-a l g e b r a}} \mathcal{G}$. To do this we first define a product on rank-one, torsion free sheaves that is compatible with the tensor product and the spin structure.

Near a geometric node $\mathfrak{p}$ of $C$ over $s$ in $S$, the strict Henselization $\mathcal{O}_{C, \mathfrak{p}}^{\text {sh }}$ is isomorphic to $A=\left(R[x, y] / x y-t^{l}\right)^{\mathrm{sh}}$ where $R$ is the strict Henselization of the base $R=\mathcal{O}_{S, s}^{\text {sh }}$. The sheaf $\mathcal{F}_{r}$ corresponds to an $A$-module $E_{u, v}:=\left\langle\xi_{1}, \xi_{2}\right| t^{v} \xi_{1}=$ $\left.x \xi_{2}, t^{u} \xi_{2}=y \xi_{1}\right\rangle$, where $u+v=l$.

For any integers $i, j, i^{\prime}, j^{\prime}$ such that $i+j=i^{\prime}+j^{\prime}=l$, we will define a product

$$
E_{i, j} \otimes E_{i^{\prime}, j^{\prime}} \rightarrow E_{\overline{i+i^{\prime}}, \overline{j+j^{\prime}}} .
$$

Here, for any integer $a$, we denote by $\bar{a}$ the smallest non-negative integer congruent to $a(\bmod l)$. Intuitively, one may think of these modules as pushforwards $\pi_{*} \mathcal{L}=$ $\left\langle z^{i} s, w^{j} s\right\rangle$ and $\pi_{*} \mathcal{L}^{\prime}=\left\langle z^{i^{\prime}} s^{\prime}, w^{j^{\prime}} s^{\prime}\right\rangle$ of invertible sheaves $\mathcal{L}$ and $\mathcal{L}^{\prime}$ on $\mathcal{C}$, and the product as the map $\pi_{*} \mathcal{L} \otimes \pi_{*} \mathcal{L}^{\prime} \rightarrow \pi_{*}\left(\mathcal{L} \otimes \mathcal{L}^{\prime}\right)$ induced by adjointness.

More precisely, we define the product as follows. First, if $i+i^{\prime}>l$, then $j+j^{\prime}<l$, $i>j^{\prime}$, and $i^{\prime}>j$. Furthermore,

$$
\overline{i+i^{\prime}}=i+i^{\prime}-l=i-j^{\prime}=i^{\prime}-j
$$

and

$$
\overline{j+j^{\prime}}=j+j^{\prime}
$$

In this case, if $E_{i, j}=\left\langle\zeta_{1}, \zeta_{2} \mid \tau^{j} \zeta_{1}=x \zeta_{2}, \tau^{i} \zeta_{2}=y \zeta_{1}\right\rangle, E_{i^{\prime} j^{\prime}}=\left\langle\xi_{1}, \xi_{2}\right| \tau^{j^{\prime}} \xi_{1}=$ $\left.x \xi_{2}, \tau^{i^{\prime}} \xi_{2}=y \xi_{2}\right\rangle$, and $E_{\overline{i+i^{\prime}}, \overline{j+j^{\prime}}}=\left\langle\nu_{1}, \nu_{2} \mid \tau^{\overline{j+j}} \nu_{1}=x \nu_{2}, \tau^{\overline{i+i^{\prime}}} \nu_{2}=y \nu_{1}\right\rangle$, then the product $E_{i, j} \otimes E_{i^{\prime}, j^{\prime}} \rightarrow E_{\overline{i+i^{\prime}}}, \overline{j+j^{\prime}}$ is defined on these generators as

$$
\zeta_{1} \otimes \xi_{1} \mapsto x \nu_{1}, \quad \zeta_{1} \otimes \xi_{2} \mapsto \tau^{j^{\prime}} \nu_{1}, \quad \zeta_{2} \otimes \xi_{1} \mapsto \tau^{j} \nu_{1}, \text { and } \quad \zeta_{2} \otimes \xi_{2} \mapsto \nu_{2}
$$

Similarly, if $i+i^{\prime}<l$, then $j+j^{\prime}>l$ and $j-i^{\prime}=j^{\prime}-i=\overline{j+j^{\prime}}$, and we define the product by

$$
\zeta_{1} \otimes \xi_{1} \mapsto \nu_{1}, \quad \zeta_{1} \otimes \xi_{2} \mapsto \tau^{i^{\prime}} \nu_{2}, \quad \zeta_{2} \otimes \xi_{1} \mapsto \tau^{i} \nu_{2}, \text { and } \quad \zeta_{2} \otimes \xi_{2} \mapsto y \nu_{2}
$$

Finally if $i+i^{\prime}=j+j^{\prime}=l$, then $\overline{i+i^{\prime}}=\overline{j+j^{\prime}}=0, i=j=i^{\prime}=j^{\prime}=l / 2$, $E_{0,0}=\langle\sigma\rangle$ is free, and the product is

$$
\zeta_{1} \otimes \xi_{1} \mapsto x \sigma, \quad \zeta_{1} \otimes \xi_{2} \mapsto \tau^{i} \sigma, \quad \zeta_{2} \otimes \xi_{1} \mapsto \tau^{i} \sigma, \quad \zeta_{2} \otimes \xi_{2} \mapsto y \sigma .
$$

Note that if $d$ is relatively prime to $l$, then for any non-free $E_{i, j}$ with $i+j=l$, the $d$ th power $E_{\overline{d i}, \overline{d j}}$ is also not free, i.e., $\overline{d i}$ and $\overline{d j}$ are non-zero. 
It is easy to see that these product maps are commutative and associative and induce the power maps of [4] in the case of $\left(E_{i, j}\right)^{\otimes d}$. They also agree with the standard tensor product on the locus where $E_{i, j}$ and $E_{i^{\prime}, j^{\prime}}$ are free. By this we mean the following: When $x$ is invertible, we have $E_{i, j}=\left\langle\zeta_{1}\right\rangle$ with $\zeta_{2}=\tau^{i} \zeta_{1} / x$, and $E_{i^{\prime}, j^{\prime}}=\left\langle\xi_{1}\right\rangle$ with $\xi_{2}=\tau^{i^{\prime}} \xi_{1} / x$. Letting $\nu_{1}=\zeta_{1} \otimes \xi_{1}$, we have an isomorphism $E_{i, j} \otimes E_{i^{\prime}, j^{\prime}} \rightarrow E_{\overline{i+i^{\prime}}, j+j^{\prime}}$. It is easy to check that this "standard tensor product" isomorphism is identical to the isomorphism induced by our product maps. When $y$ is invertible, a similar calculation shows the power maps again agree with the standard tensor product, and, of course, these are all compatible when $x$ and $y$ are both invertible.

This local product map allows us to define our $\mathcal{O}_{C}$-algebra $\mathcal{G}$ from an $r$-spin structure $\left(\left\{\mathcal{F}_{r}\right\},\left\{c_{d, d^{\prime}}\right\}\right)$ on $C$ as follows. Let $\mathcal{G}_{0}:=\mathcal{O}_{C}$, and for each positive divisor $d$ of $r$ let $\mathcal{G}_{d}:=\mathcal{F}_{r / d}$, and $\mathcal{G}_{d+n r}:=\left(\omega_{\text {log }}^{C}\right)^{\otimes n} \otimes \mathcal{F}_{r / d}$. For all remaining indices $d$, if $e=\operatorname{gcd}(d, r)$ and $e \neq d$, then on the open locus where $\mathcal{G}_{e}$ is locally free, let $\mathcal{G}_{d}=\mathcal{G}_{e}^{\otimes d / e}$. At each node $\mathfrak{p}$ where $\mathcal{G}_{e}$ is not locally free, the local structure is $C^{\mathrm{sh}} \cong \operatorname{Spec}\left(R[x, y] / x y-t^{l}\right)^{\mathrm{sh}}$, with $l$ a divisor of $r$, and we have an isomorphism $\alpha: \mathcal{G}_{1}^{\text {sh }} \stackrel{\sim}{\rightarrow} E_{i, j}$, where $i=i_{r}, j=j_{r}$, and $i+j=l$. Further, $\alpha$ induces an isomorphism $\mathcal{G}_{e}^{\mathrm{sh}} \cong E_{\overline{e i}}, \overline{e j}$. We let $\mathcal{G}_{d}^{\mathrm{sh}}:=E_{\overline{d i}}, \overline{d j}$ and use the gluing induced by the power maps $\left(E_{i, j}^{\otimes d}\right) \rightarrow\left(E_{\overline{e i}, \overline{e j}}\right)^{\otimes d / e} \rightarrow E_{\overline{d i}}, \overline{d j}$ and by the tensor product $\left.\mathcal{G}_{1}\right|_{C-\mathfrak{p}} ^{\otimes d} \cong$ $\left.\left.\mathcal{G}_{e}\right|_{C-\mathfrak{p}} ^{\otimes d / e} \cong \mathcal{G}_{d}\right|_{C-\mathfrak{p}}$. These are compatible, as discussed above. This gives us $\mathcal{G}_{e}$ for every $e \geq 0$.

The dual $\left(E_{i, j}\right)^{\vee}$ is canonically isomorphic to $E_{j, i}$ and thus we also may define $\mathcal{G}_{-d}=\mathcal{G}_{d}^{\vee}$ and extend the $\mathcal{O}_{C}$-algebra structure to

$$
\mathcal{G}:=\bigoplus_{d \in \mathbb{Z}} \mathcal{G}_{d}
$$

There is, however, a potential ambiguity in the gluing in the construction of the $\mathcal{G}_{e}$, arising from the choice of isomorphism $\alpha: \mathcal{G}_{1}^{\text {sh }} \stackrel{\sim}{\rightarrow} E_{i, j}$. The following lemma shows that this is not a problem.

Lemma 4.4.1. Given any two isomorphisms $\alpha: \mathcal{G}_{1}^{\text {sh }} \stackrel{\sim}{\rightarrow} E_{i, j}$ and $\beta: \mathcal{G}_{1}^{\text {sh }} \stackrel{\sim}{\rightarrow} E_{i, j}$, the two sheaves of algebras, as constructed above, are isomorphic.

Proof. Let $\mathcal{G}_{d}^{\alpha}$ and $\mathcal{G}_{d}^{\beta}$ be the two sheaves induced from the two choices of isomorphism. First, if the automorphism $\alpha^{-1} \beta$ is given by multiplication by an element of $A^{\times}$, then $\mathcal{G}_{d}^{\alpha}$ and $\mathcal{G}_{d}^{\beta}$ are clearly isomorphic (the difference in gluing data is a Cech coboundary). Consequently, we may assume that for each $e$ dividing $r$ and such that $\mathcal{G}_{e}$ is locally free at $\mathfrak{p}$, i.e., ei and $e j$ are congruent to $0(\bmod l)$, an isomorphism $\mathcal{G}_{e} \stackrel{\sim}{\rightarrow} E_{0,0} \cong A$ has been fixed, and the automorphism $\beta \alpha^{-1}$ preserves the homomorphisms

$$
\gamma_{e}: E_{i, j} \rightarrow E_{\overline{e i}}, \overline{e j}=E_{0,0}
$$

induced from $c_{r, r / e}: \mathcal{G}_{1}^{\otimes e}=\mathcal{E}_{r}^{\otimes e} \rightarrow \mathcal{E}_{r / e}=\mathcal{G}_{e}$.

It is straightforward to see [5. Prop. 4.2.12] that the automorphisms of $E_{i, j}$ that preserve $\gamma_{e}$ are elements of $\boldsymbol{\mu}_{e} \times \boldsymbol{\mu}_{e}$, acting on $E_{i, j}=\left\langle\xi_{1}, \xi_{2} \mid x \xi_{2}=t^{i} \xi_{1}, y \xi_{1}=t^{i} \xi_{2}\right\rangle$ in the obvious way. Moreover, if either $t^{i}$ or $t^{j}$ is not zero, or if $t^{i}$ and $t^{j}$ are zero, but the normalization of $C$ at $\mathfrak{p}$ is connected, then automorphisms of $E_{i, j}$ preserving 
$\gamma_{e}$ must, in fact, lie in the image of the diagonal $\boldsymbol{\mu}_{e} \hookrightarrow \boldsymbol{\mu}_{e} \times \boldsymbol{\mu}_{e}$. Thus they are all induced by multiplication by elements of $A^{\times}$, and consequently induce isomorphic sheaves: $\mathcal{G}_{d}^{\alpha} \stackrel{\sim}{\rightarrow} \mathcal{G}_{d}^{\beta}$.

Therefore, the only potential ambiguity arises when $t^{i}=t^{j}=0$, the normalization of $C$ at $\mathfrak{p}$ is disconnected, and $\beta \alpha^{-1}$ corresponds to an element $(\eta, \sigma)$ of $\boldsymbol{\mu}_{r} \times \boldsymbol{\mu}_{r}$. In this case, we have $t^{l}=t^{i+j}=0$, and $A=(R[x, y] / x y)^{\text {sh }}$, and thus $E_{i, j}=\left\langle\xi_{1}, \xi_{2} \mid x \xi_{2}=y \xi_{1}=0\right\rangle$. Likewise, since $\overline{d i}$ and $\overline{d j}$ are non-zero, we have $E_{\overline{d i}, \overline{d j}}=\left\langle\zeta_{1}, \zeta_{2} \mid x \zeta_{2}=y \zeta_{1}=0\right\rangle$. But now the automorphisms $\eta^{d}$ and $\sigma^{d}$ applied to $\left.\mathcal{G}_{d}\right|_{C-\mathfrak{p}}$ on the two connected components of $C-\mathfrak{p}$, respectively, give an isomorphism $\mathcal{G}_{d}^{\alpha} \stackrel{\sim}{\rightarrow} \mathcal{G}_{d}^{\beta}$, as desired.

Since the sheaves $\mathcal{E}_{i}$ in the $r$-spin structure are (flat) rank-one, torsion free sheaves with $\mathcal{E}_{1}=\mathcal{G}_{r}=\omega_{\text {log }}$, the scheme

$$
P_{\mathcal{G}}:=\operatorname{Spec}_{\mathcal{O}_{C}}(\mathcal{G})
$$

is an object of $\mathcal{P}_{g, n}\left(\omega_{l o g}^{1 / r}\right)$, and it is straightforward to see that this functor

$$
\overline{\mathcal{M}}_{g, n}^{1 / r} \rightarrow \mathcal{P}_{g, n}\left(\omega_{\text {log }}^{1 / r}\right)
$$

is the inverse of the composite

$$
\mathcal{P}_{g, n}\left(\omega_{l o g}^{1 / r}\right) \rightarrow \mathcal{B}_{g, n}\left(\mathbb{G}_{\mathbf{m}}, \omega_{l o g}^{1 / r}\right) \rightarrow \overline{\mathcal{M}}_{g, n}^{1 / r}
$$

\section{A fEW REMARKS ON ANALYTIC TWISTED $r$-SPIN CURVES, WiTTEN'S CLASS, AND THE $\bar{\partial}$-OPERATOR}

The stack of $r$-spin curves is interesting primarily because of the role it plays in the generalized Witten conjecture [1], relating intersection theory on $\overline{\mathcal{M}}_{g, n}^{1 / r}$ to the Gelfand-Dikii $\left(K d V_{r}\right)$ hierarchy. This conjecture depends on the construction of a virtual Euler class $c(\mathcal{V})$ for the pushforward of the universal $r$-spin bundle. That is, if $q: \mathcal{D} \rightarrow \overline{\mathcal{M}}_{g, n}^{1 / r}$ is the universal curve over $\overline{\mathcal{M}}_{g, n}^{1 / r}$, and if $\mathcal{F}_{r}$ is the universal $r$ th root bundle (from the universal coherent net) on $\mathcal{D}$, then $c(\mathcal{V})$ is a virtual Euler class for $\mathcal{V}:=R q_{*} \mathcal{F}_{r}$.

In particular, when $R^{0} q_{*} \mathcal{F}_{r}=0$, then $c(\mathcal{V})$ is just the Euler class of $-R^{1} q_{*} \mathcal{F}_{r}$, but in general $c(\mathcal{V})$ is not the Euler class of the index bundle $R q_{*} \mathcal{F}_{r}$, even when such a class is well defined.

We now describe how to extend Witten's construction to twisted $r$-spin curves.

5.1. The $\bar{\partial}$-operator. Witten's construction of $c(\mathcal{V})$ depends on extending the operator $\bar{\partial}: \Omega^{0,0}\left(\mathcal{F}_{r}\right) \rightarrow \Omega^{0,1}\left(\mathcal{F}_{r}\right)$ to nodal curves; that is, to the boundary of $\overline{\mathcal{M}}_{g, n}^{1 / r}$. This is done by Seeley and Singer in [10] for $r=2$, and the construction for general $r$ is similar. Details of the general construction are given in 8]. This construction applies as well in the case of twisted $r$-spin curves.

Theorem 5.1.1. For a family of twisted curves $\left(\mathcal{C} / S, \mathcal{S}_{i}^{e}\right)$ and for any line bundle $\mathcal{L}$ on $\mathcal{C}$, there is a continuous family of Fredholm operators $\bar{\partial}: \Omega^{0,0}(\mathcal{L}) \rightarrow \Omega^{0,1}(\mathcal{L})$ from $\mathcal{L}$-valued $(0,0)$-forms to $\mathcal{L}$-valued $(0,1)$-forms. Moreover, if $\pi: \mathcal{C} \rightarrow C$ is the coarse moduli space of $\mathcal{C}$, then $\bar{\partial}$ induces the $\bar{\partial}$ operator of Seeley and Singer on the pushforward to $C$ :

$$
\bar{\partial}: \Omega^{0,0}\left(\pi_{*} \mathcal{L}\right) \rightarrow \Omega^{0,1}\left(\pi_{*} \mathcal{L}\right)
$$


Proof. Near a point $p \in \mathcal{C}$ we have $\mathcal{C}^{\text {sh }}=\left[U / \boldsymbol{\mu}_{l}\right]$. Seeley and Singer's local description of $\bar{\partial}$ clearly applies to any line bundle on $U$. Moreover $\bar{\partial}$ is $\boldsymbol{\mu}_{l}$-equivariant, and thus passes to $\mathcal{C}$, as well as inducing the usual $\bar{\partial}$ operator on the coarse moduli C.

5.2. Witten's class. Witten's construction of the virtual class $c(\mathcal{V})$ on $\overline{\mathcal{M}}_{g, n}^{1 / r}$ works almost verbatim for $\mathcal{B}_{g, n}\left(\mathbb{G}_{\mathbf{m}}, \omega_{\text {log }}^{1 / r}\right)$, where his sheaf $\mathcal{T}$ (our $\mathcal{F}_{r}$ ) on the universal curve over $\overline{\mathcal{M}}_{g, n}^{1 / r}$ is replaced by the universal $r$ th-root line bundle $\mathcal{L}$ on the universal curve over $\mathcal{B}_{g, n}\left(\mathbb{G}_{\mathbf{m}}, \omega_{\text {log }}^{1 / r}\right)$.

The only other difference is the fact that $\pi_{*} \mathcal{L}$ is an $r$ th root of $\omega_{l o g}\left(-\sum m_{i} p_{i}\right)$, rather than an $r$ th root of $\omega\left(-\sum m_{i} p_{i}\right)$.

In terms of our construction, that means that each $m_{i}$, or rather the local index of the twisted curve $\mathcal{C}$ near each canonical section $\mathcal{S}_{i}$, must be at least 1 to make the Witten construction work. In the case that the local index near any $\mathcal{S}_{i}$ is zero, we define the class $c(\mathcal{V})$ to be 0 . This is compatible with the axioms of [7] (and the axiom of descent [6]), provided those axioms hold for the Witten class when the local index at each $\mathcal{S}_{i}$ is greater than 0 .

An algebraic construction of Witten's class has recently been given by Polishchuk and Vaintrob in 9]. Their construction should also work for twisted spin curves, since the sheaves involved in that construction are all pushforwards (to the base of the family) of sheaves induced on the coarse moduli $C$ by pushing forward tautological sheaves from the twisted curve $\mathcal{C}$ to $C$.

\section{ACKNOWLEDGEMENTS}

We heartily thank Angelo Vistoli and the referee for helpful comments and Heidi Jarvis for help with typesetting.

\section{REFERENCES}

[1] D. Abramovich and A. Vistoli, Compactifying the Space of Stable Maps, J. Amer. Math. Soc. 15 (2002), 27-75.

[2] D. Abramovich, A. Corti and A. Vistoli, Twisted bundles and admissible covers, preprint (2001), math.AG/0106211.

[3] P. Deligne and D. Mumford, The Irreducibility of the Space of Curves of Given Genus, Inst. Hautes Études Sci. Publ. Math. No. 36 (1969), 75-109. MR 41:6850

[4] T. Jarvis, Torsion-Free Sheaves and Moduli of Generalized Spin Curves, Compositio Math. 110 (1998), no. 3, 291-333. MR 99b:14026

[5] T. Jarvis, Geometry of the moduli of higher spin curves, Internat. J. of Math. 11 (2000), no. 5, 637-663. MR 2001f:14050

[6] T. Jarvis, T. Kimura, and A. Vaintrob, Gravitational Descendants and the Moduli Space of Higher Spin Curves. In E. Previato (Ed.), Advances in Algebraic Geometry Motivated by Physics (Lowell, MA, 2000), 167-177, Contemp. Math., 276, Amer. Math. Soc., Providence, RI, 2001.

[7] T. Jarvis, T. Kimura and A. Vaintrob, Moduli spaces of higher spin curves and integrable hierarchies. Compositio Math., 126 (2001), no. 2, 157-212.

[8] T. Mochizuki, The Virtual Class of the Moduli Stack of r-spin Curves, preprint, (2001). http://math01.sci.osaka-cu.ac.jp/ takuro/list.html

[9] A. Polishchuk and A. Vaintrob Algebraic Construction of Witten's Top Chern Class. In E. Previato (Ed.), Advances in Algebraic Geometry Motivated by Physics (Lowell, MA, 2000), 229-249, Contemp. Math., 276, Amer. Math. Soc., Providence, RI, 2001. 
[10] R. Seeley and I. M. Singer, Extending $\bar{\partial}$ to Singular Riemann Surfaces, J. Geom. Phys 5 (1988), no.1, 121-136. MR 91f:58101

[11] E. Witten, Algebraic Geometry Associated with Matrix Models of Two-dimensional Gravity, Topological models in modern mathematics (Stony Brook, NY, 1991), Publish or Perish, Houston, TX 1993, 235-269. MR 94c:32012

Department of Mathematics, Boston University, 111 Cummington Street, Boston, MASSACHUSETTS 02215

E-mail address: abrmovic@math.bu.edu

Department of Mathematics, Brigham Young University, Provo, Utah 84602

E-mail address: jarvis@math.byu.edu 